\title{
Evaluación de tecnologías sanitarias: Dimero $D$ por inmunoensayo como marcador biológico de Tromboembolismo Venoso
}

Inmunoassay D-Dimer Test as Marker of Venous Thromboembolism

Cortesía del Instituto de Efectividad Clínica y Sanitaria: www.iecs.org.ar

\section{Introducción}

El tromboembolismo venoso (TEV) incluye la trombosis venosa profunda de los miembros inferiores (TVP) y el tromboembolismo de pulmón (TEP). Se estima una incidencia anual de TEV de 1 a 2/1000 personas, correspondiendo dos tercios a TVP y el tercio restante a TEP. En la actualidad el TEP presenta dificultades para su diagnóstico y ante su sospecha, el dímero $\mathrm{D}(\mathrm{DD})$ ha sido propuesto como una evaluación no invasiva, como económica, rápida y simple.

Por ejemplo, y siguiendo las recomendaciones de los investigadores del estudio PIOPED II (Prospective investigation of Pulmonary Embolism Diagnosis II) si la probabilidad clínica de TEP es baja o moderada y los resultados del DD son negativos, podría excluirse la presencia de TEV, debiéndose realizar otras evaluaciones independientes del resultado del DD si la probabilidad clínica es alta. Ver figura 1.

Figura 1: algoritmo que sintetiza el abordaje diagnóstico de los pacientes con sospecha de tromboembolismo pulmonar (TEP) de acuerdo a la fuerza de la sospecha clínica de tromboembolismo venoso (TEV).

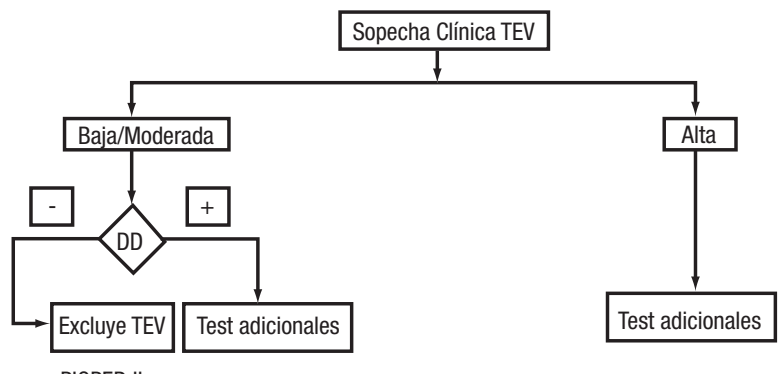

PIOPED ॥

Fuente: Stein, P. Diagnostic pathways in acute pulmonary embolism: recommendations of the PIOPED II investigators. The American Journal of Medicine, 2006; 119: p. 1048.

\section{La tecnología}

El dímero $D$ es un producto de la degradación de la fibrina que puede ser detectado por técnicas cualitativas o cuantitativas. Estas técnicas tienen diferentes tiempos de procesamiento. En este documento se evaluarán aquellas técnicas cuantitativas rápidas (15 a 35 minutos) existentes en el mercado: ELISA fluorescente (ELFA) y el látex por inmunoturbidimetría.

\section{Objetivo}

El objetivo del presente informe es evaluar la utilidad del DD por inmunoensayo para la detección del TEV.

\section{Métodos}

Se realizó una búsqueda en las principales bases de datos bibliográficas (MEDLINE, EMBASE, Cochrane, DARE, NHS
EED) en buscadores genéricos de Internet, agencias de evaluación de tecnologías sanitarias y financiadores de salud. Se priorizó la inclusión de revisiones sistemáticas, ensayos clínicos controlados aleatorizados (ECA) evaluaciones de tecnologías sanitarias y económicas, guías de práctica clínica y políticas de cobertura de otros sistemas de salud.

\section{Resultados}

Se encontraron cinco metanálisis ${ }^{2,3,4,5,6}$ (que incluyeron estudios publicados entre 1980 y 2005), una serie de casos, una evaluación de tecnología sanitaria (ETS) y un documento de un financiador de salud estadounidense que evaluaron la precisión diagnóstica de los test de DD para la exclusión del TEV. Se resumen a continuación sus principales resultados.

\section{Para trombosis venosa profunda}

Dos de ellos reportaron una sensibilidad (S) del ELFA de $96 \%$ (IC95\% 90 a 100\%) y una razón de verosimilitud negativa (del inglés: Likelihood ratio: LR) de 0,09 $(0,02$ a 0,41). Uno de ellos reportó una discreta superioridad del ELFA (S: 93\%; 89 a 95) sobre el látex (S: 85\%; 78 a 94).

\section{Para tromboembolismo pulmonar}

Tanto el ELFA como el látex presentaron elevadas sensibilidades (97\%; 88 a 99$)$ y buenos LR negativos $(0,07 ; 88$ a 98$)$ y 0,10 , respectivamente. En pacientes con baja o moderada probabilidad de TEP, el LR negativo de un DD menor a 500 $\mathrm{mcg} / \mathrm{L}$ fue comparable a las técnicas habitualmente utilizadas, con una probabilidad post-test negativo menor a $5 \%$.

\section{Costo}

En Argentina el costo promedio de una determinación de DD por ELFA es de u\$s 11 y por látex inmunoturbidimétrico de u\$s 9 (precios de enero de 2009).

\section{Conclusiones}

EI DD es una evaluación no invasiva, rápida, económica y segura. En pacientes con baja o moderada probabilidad clínica, los inmunoensayos cuantitativos rápidos de DD demostraron elevada capacidad para la exclusión del TEV, siendo la misma comparable a la de algunos de los estudios por imágenes.

Resumido de: Pichon-Riviere A, Augustovski F, Aruj P, Bardach A, Calcagno J, Galante J, García Martí S, Glujovsky D, Linetzky B, López A, Regueiro A. Dimero D por inmunoensayo como marcador biológico de Tromboembolismo Venoso. Documentos de Evaluación de Tecnologías Sanitarias, Informe de Respuesta Rápida № 163, Buenos Aires, enero de 2009.

\section{Referencia}

1. Stein, P. Diagnostic pathways in acute pulmonary embolism: recommendations of the PIOPED II investigators. The American Journal of Medicine, 2006; 119 : p. 1048. 2. Di Nisio, M. Diagnostic accuracy of D-dimer test for exclusion of venous thromboembolism: a systematic review. Journal of thrombosis and haemostasis, $2007 ; 5$ : p. 296. 3. Stein, P. D-dimer for the exclusion of acute venous thrombosis and pulmonary embolism. A systematic review. Annals of Internal Medicine, 2004. 140: p. 589. 4. Roy, P. Systematic review and meta-analysis of strategies for the diagnosis of suspected pulmonary embolism. BMJ, 2005. 331: p. 1. 5. Brown, M. The accuracy of the enzyme-linked immunosorbent assay D-Dimer Test in the diagnosis of pulmonary embolism: A meta-analysis. Ann. of emergency medicine. 2002; 40: p.133.

6. Brown M. Turbidimetric D-Dimer Test in the diagnosis of pulmonary embolism: A meta-analysis. Clinical Chemistry. $2003 ; 49$ (11): p.1846. 